\title{
Characteristics of Planar Monopole Antenna on High Impedance Electromagnetic Surface
}

\author{
Maximilian C. Scardelletti ${ }^{1}$, Nathan Jastram ${ }^{1,2}$, George E. Ponchak ${ }^{1}$ and Rhonda R. Franklin ${ }^{2}$ \\ 1. NASA Glenn Research Center, Cleveland, Ohio, MS: 54-5, 44135 \\ 2. University of Minnesota, Minneapolis, Minnesota
}

\begin{abstract}
This paper presents for the first time measured characteristics of a planar monopole antenna placed directly on a high impedance electromagnetic surface or artificial magnetic conductor (AMC). The return loss and radiation patterns are compared between the antenna in free space, and when placed directly on a perfect electrical conductor (PEC), and on the AMC. The antenna measured in free space has a wide pass band from 3 to $10 \mathrm{GHz}$. The return loss for the antenna on the PEC is nearly all reflected back and the return loss for the antenna on the AMC has a $10 \mathrm{~dB}$ bandwidth from 7.5 to $9.5 \mathrm{GHz}$. The gain of the antenna in free space, on PEC and on AMC is 1,-12 and 10 $\mathrm{dBi}$, respectively. This indicates that the $A M C$ is working properly, sending all the radiation outward with little loss.
\end{abstract}

Index Terms - Antenna, Artificial Magnetic Conductor, High Impedance Electromagnetic Surface.

\section{INTRODUCTION}

In many applications, it is required to place an antenna directly onto a large, perfectly electrically conducting metal structure. For example, wireless sensors or wireless communication devices may need to be placed on the fuselage of an airplane, on the side of a machine, or within or on an automobile. In these cases, the antenna must still provide good radiation characteristics, but the large metal structures will degrade the radiation characteristics of many types of antennas.

Sievenpiper developed and demonstrated highimpedance electromagnetic surfaces or artificial magnetic conductors (AMC) [1]-[3] to enable antennas to be placed near perfectly electrically conducting (PEC) surfaces. For example, a wire antenna placed horizontally over an AMC was demonstrated to have improved gain compared to the same antenna over a PEC [2], [4]. Subsequent papers further developed the technology. A microstrip patch antenna with an AMC ground plane and a partially reflective surface placed a quarter-wavelength from the antenna substrate has good gain [5], and this technology was expanded to create a dual-band antenna [6]. However, these two antennas required a superstrate, which increases the antenna complexity and thickness. A slot antenna over a PEC with an AMC structure between them was demonstrated to improve antenna performance by reducing surface wave modes [7]. A $60 \mathrm{GHz}$ antenna on an AMC was demonstrated for on-chip receivers [8], and a wide-bandwidth, planar monopole has been simulated on an AMC surface [9]. These last two papers are thin, low profile antennas with the antenna placed in very close proximity to the $\mathrm{AMC}$, which is required for many applications.

In this paper, we report the first measured characteristics of a planar, monopole antenna placed in close proximity to an AMC surface. The return loss and radiation characteristics are compared between the antenna in free space, placed directly on a PEC, and placed directly on an AMC, separated only by the $100 \mu \mathrm{m}$ thickness of the substrate.

\section{ANTENNA AND AMC DESCRIPTION}

The antenna is an ultra-wideband (UWB), planar monopole antenna built on $100 \mu \mathrm{m}$ thick LCP [10], [11]. This antenna has the advantage of operating from 3 to 10 $\mathrm{GHz}$ and is fed by a coplanar waveguide (CPW) line, which minimizes radiation from the feed line. An SMA connector was soldered to the CPW feed line to facilitate measurement.

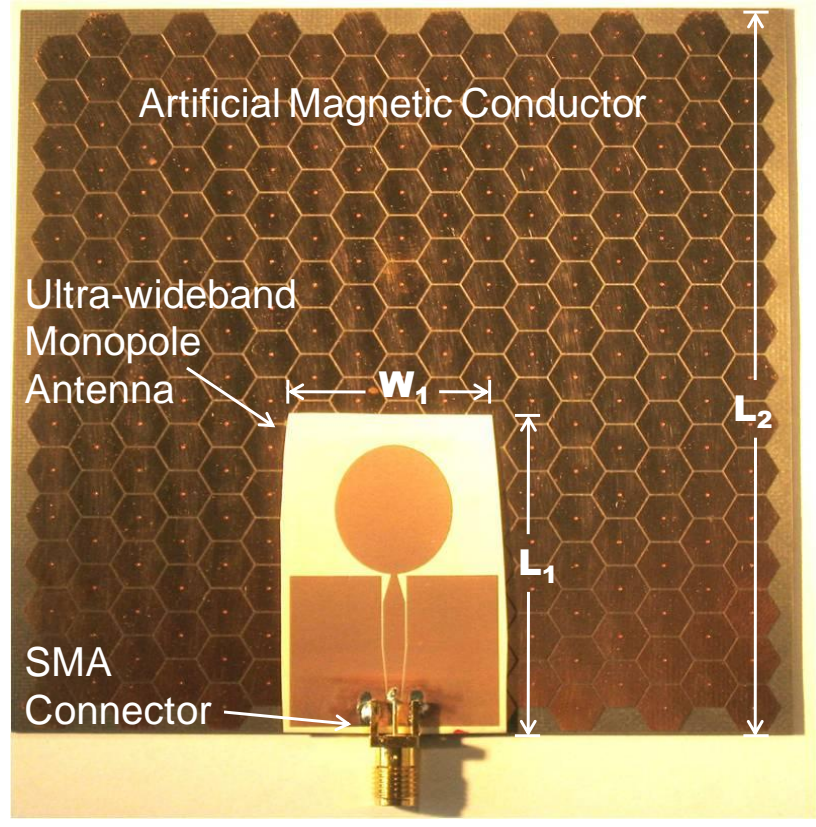

Figure 1. Ultra-wideband monopole antenna on artificial magnetic conductor (AMC). 
There are many AMC structures described in the literature. Here, the hexagonal mushroom-like structure first described in [1]-[3] is used. The AMC structure is fabricated on a piece of $1.01 \mathrm{~mm}$ thick RT Duriod, $\varepsilon r=2.2$. It is shown in Fig. 1 along with the ultra-wideband planar monopole antenna. Note that the antenna is placed directly on top of the AMC only separated by the LCP thickness. The dimensions of the hexagonal mushroom structures were calculated using equations in [3] for a $10 \mathrm{GHz}$ center frequency and are illustrated in Fig. 2. The dimensions for $\boldsymbol{W}_{\boldsymbol{1}}, \boldsymbol{L}_{\boldsymbol{1}}$ and $\boldsymbol{L}_{2}$ are 29, 43.5 and $100 \mathrm{~mm}$, respectively. Note the width of the AMC board is equal to its length, $100 \mathrm{~mm}$. Where $\boldsymbol{a}$ is the period distance from the center of the via of a hexagonal mushroom structure to the center of the via to an adjacent hexagonal mushroom structure, $w$ is the length of one side of the hexagon, $g$ is the gap between hexagons, $\boldsymbol{d}$ is the diameter of the via and $\boldsymbol{t}$ is the thickness of the AMC substrate. The dimensions for $\boldsymbol{a}, \boldsymbol{w}, \boldsymbol{g}, \boldsymbol{d}$, and $\boldsymbol{t}$ are $6.35,3.61,0.254,0.38$, and $1.01 \mathrm{~mm}$, respectively.

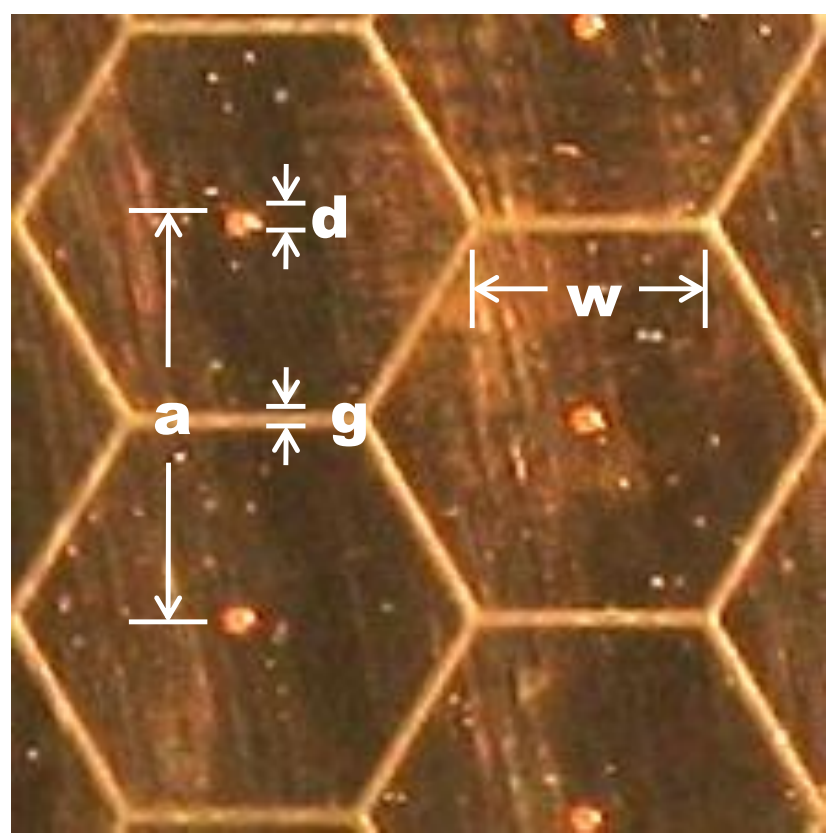

Figure 2. Hexigonal mushroom-like AMC top view.

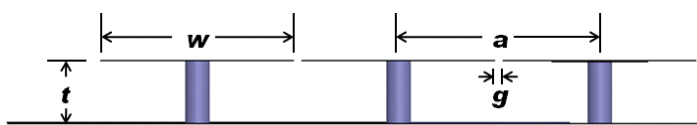

Figure 3. Side view of hexagonal AMC.

\section{ARTIFICIAL MAGNETIC CONDUCTOR/ANTENNA CHARACTERIZATION}

The reflection phase of the AMC was measured with two 2 to $18 \mathrm{GHz}$ broadband horn antennas and an Agilent E8364B Precision Network Analyzer (PNA). The two broadband horn antennas are placed next to one another facing the surface under test. The horns are only separated by a piece of microwave absorbing foam to prevent coupling between the antennas. A reference measurement is taken of a PEC surface of the same size as the AMC surface. The reflected phase of the AMC surface is divided by the reflected phase of the reference measurement and a factor of $180^{\circ}$ is added to account for the PEC having a reflection phase of $180^{\circ}$.

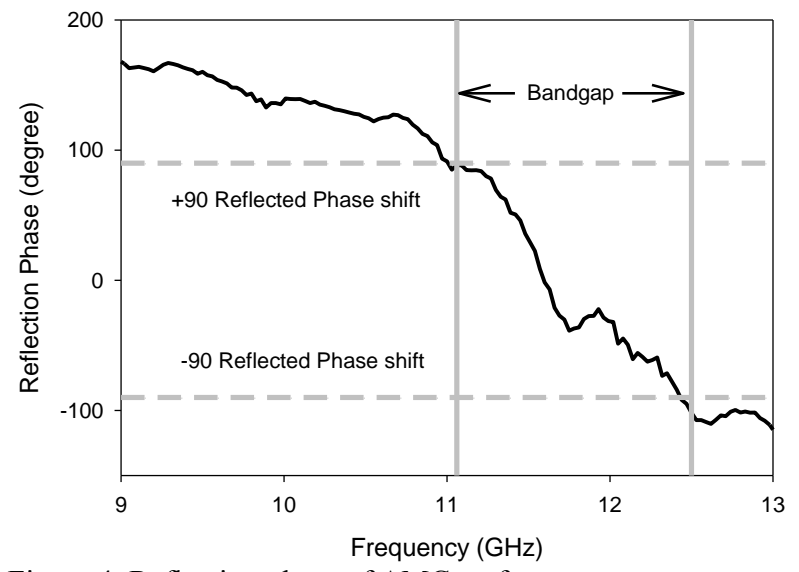

Figure 4. Reflection phase of AMC surface.

Figure 4. shows the bandgap of the AMC to be 11.06 to $12.05 \mathrm{GHz}$, defined by $90^{\circ}$ and $-90^{\circ}$ reflected phase, with the resonance of the structure at $11.63 \mathrm{GHz}$. In the bandgap, plane waves are reflected in-phase rather than out-of-phase as occurs on metal surfaces. The measured center frequency of the AMC is slightly higher than the 10 GHZ design frequency due to fabrication tolerances and approximation made in the design equations.

The return loss (RL) was measured for the UWB monopole antenna in free space, directly on a PEC and directly on an AMC, and the results are shown in Fig. 5. The antenna and structures were measured with a PNA and a HP 85052B calibration kit was used to calibrate the measurement to SMA connector soldered to the UWB antenna. The RL for the UWB in free space has a $10 \mathrm{~dB}$ bandwidth $(\mathrm{BW})$ from 3 to $10 \mathrm{GHz}$. When the antenna was measured on the PEC (ground plane only), the reflection coefficient is nearly one, indicating that currents on the ground plane cancel the currents on the antenna resulting in poor antenna characteristics. The RL for the antenna measured directly on the AMC has a $10 \mathrm{~dB}$ BW from 7.5 to $9.5 \mathrm{GHz}$. The center frequency of the antenna on the AMC does not correspond with the center frequency of the AMC shown in Fig. 4. The LCP substrate and the large metal surfaces of the antenna change the capacitance between the hexagonal structures, which affects the center frequency and bandwidth of the AMC surface. 


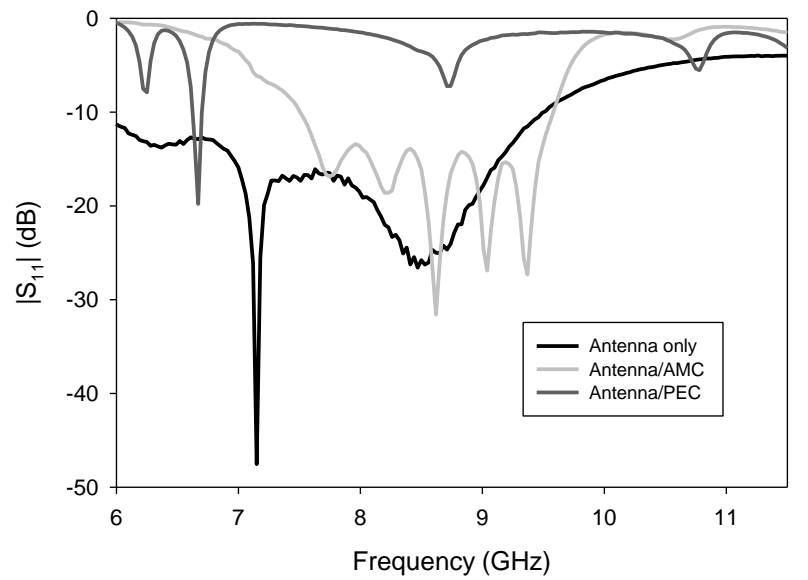

Figure 5. Measured return loss of UWB antenna in free space, antenna on PEC and antenna on AMC.

The radiation patterns for the UWB antenna in free space, on PEC and on the AMC were measured in an anechoic chamber and are shown in Figs. 6a to d. All the test equipment is outside the chamber to minimize interference from the equipment. A 15 meter cable is used to connect the test antenna to equipment. The antenna is positioned on a Styrofoam chuck and placed on a rotational stage so that a $360^{\circ}$ radiation pattern can be measured. The UWB antenna received transmitted power from a $2-18 \mathrm{GHz}$ broadband horn antenna. The UWB antenna and broadband transmit antenna are separated by a distance of $200 \mathrm{~cm}$. A Labview program is used to control the rotational stage and record the transmitted signal. The gain of the UWB antenna in free space, on PEC and on the AMC was measured using the substitution method. The radiation patterns were characterized at 8.5 $\mathrm{GHz}$, which is the center frequency of the antenna on the AMC.

The E co-pol radiation patterns of the antenna in free space, on PEC and on AMC are shown Fig. 6a. The antenna measured in free space behaves as a typical monopole antenna with a bi-directional pattern. It has gain of approximately $1 \mathrm{dBi}$. The received power of the antenna on PEC is $15 \mathrm{~dB}$ lower than the antenna measured in free space and has little backside radiation. The E copol pattern for the UWB antenna on the AMC exhibits a front side gain of $9.5 \mathrm{dBi}$ with backside radiation level similar to the antenna on PEC, indicating that the AMC surface does not support propagation currents and reflects electromagnetic waves with no phase reversal.

The $\mathrm{H}$ co-pol radiation patterns are shown in Fig. 6b and the results are similar to the E co-pol patterns. The $\mathrm{H}$ co-pol gain for the antenna in free space, on PEC and on AMC is 3, -10 and $7 \mathrm{dBi}$. Figs. $6 \mathrm{c}$ and $6 \mathrm{~d}$ are the $\mathrm{E}$ and $\mathrm{H}$ cross-pol radiation patterns. Both the $\mathrm{E}$ and the $\mathrm{H}$ cross- pol patterns are at least $15 \mathrm{~dB}$ lower than the co-pol patterns.

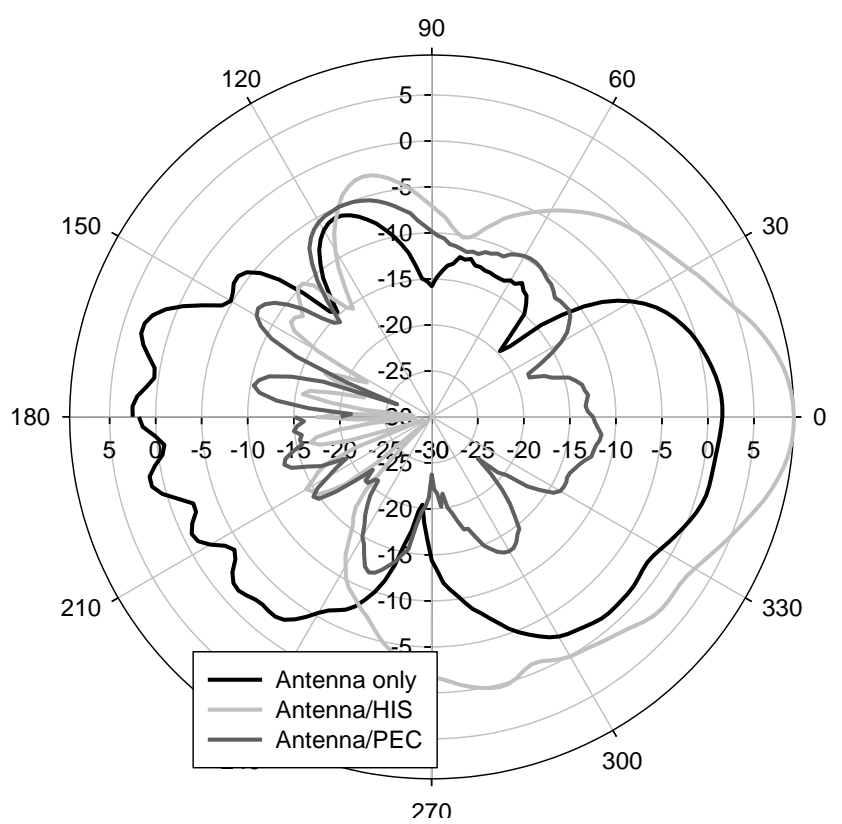

Figure 6a. E co-pol radiation pattern of antenna in free space, on $\mathrm{PEC}$ and on AMC.

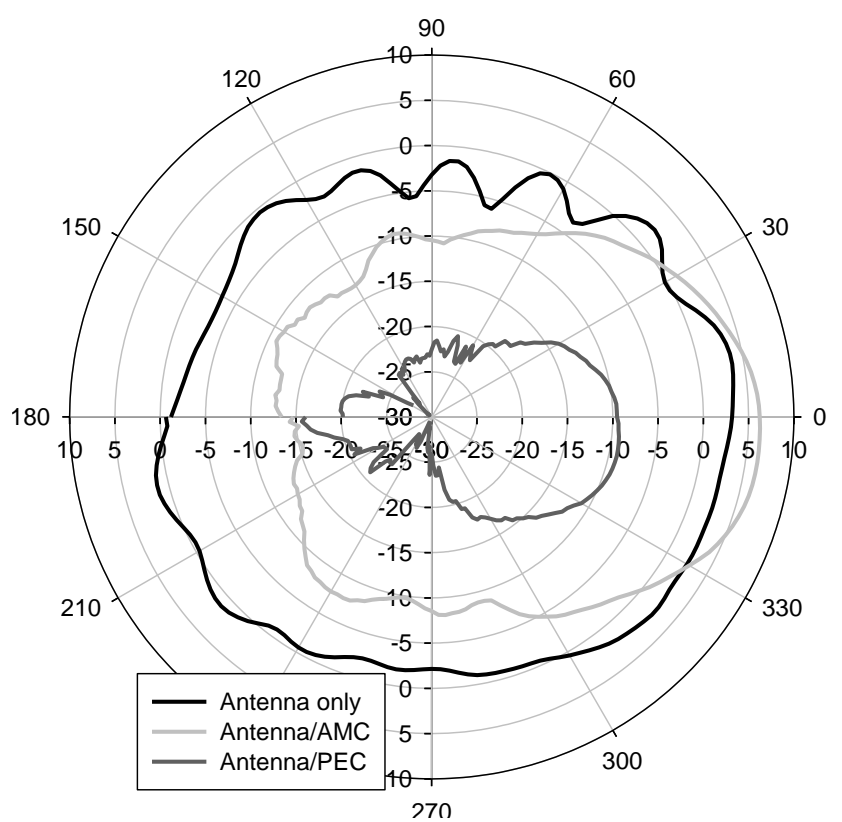

Figure $6 \mathrm{~b}$. $\mathrm{H}$ co-pol radiation pattern of antenna in free space, on PEC and on AMC. 


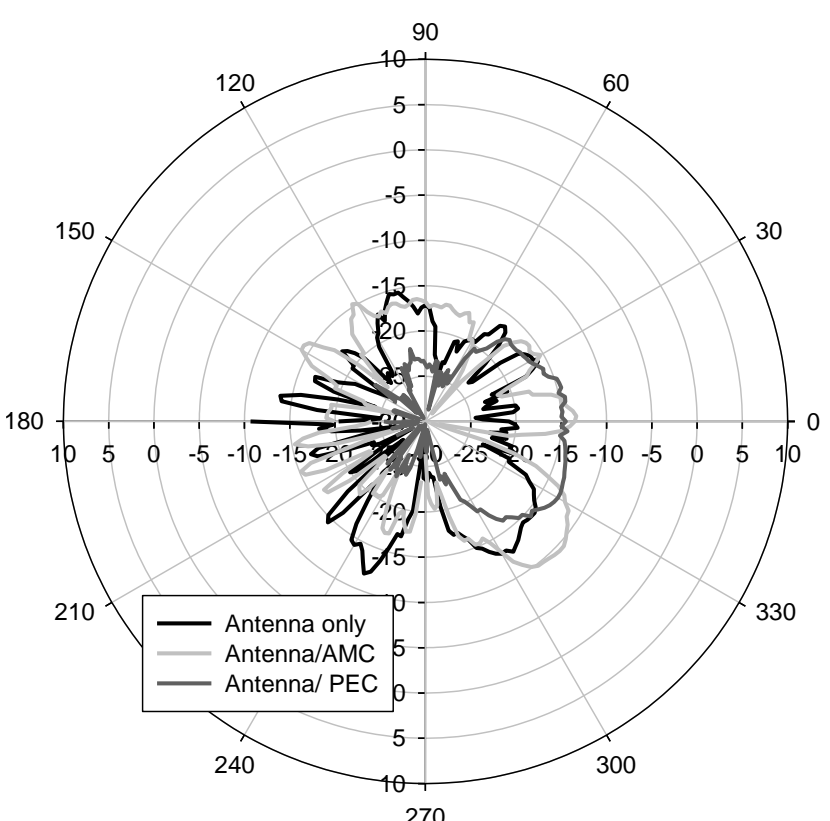

Figure $6 c$. E cross-pol radiation pattern of antenna in free space, on PEC and on AMC.

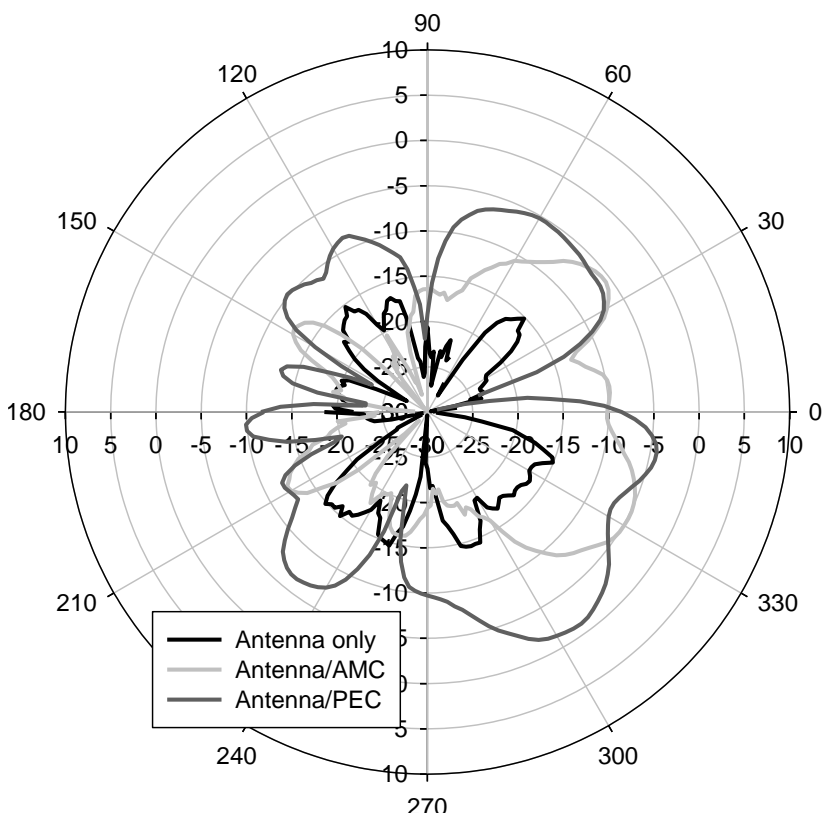

Figure $6 \mathrm{~d}$. $\mathrm{H}$ cross-pol radiation pattern of antenna in free space, on PEC and on AMC.

\section{CONCLUSION}

The measured characterization of an ultra wideband antenna on an artificial magnetic conductor has been presented for the first time. The return loss and radiation patterns are presented for the antenna in free space, placed directly on a perfect electric conductor and placed directly on an artificial magnetic conductor. The antenna when placed directly on the $\mathrm{AMC}$ has a $10 \mathrm{~dB}$ BW from 7.5 to $9.5 \mathrm{GHz}$ and a gain of $9.5 \mathrm{dBi}$, compared to a gain of 1
$\mathrm{dBi}$ and $-10 \mathrm{dBi}$ for the antenna in free space and antenna on PEC, respectively. Thus, the AMC acts as an effective shield that minimizes the effects of placing the antenna on a PEC surface. Because of the low profile of this antenna, less than $1.5 \mathrm{~mm}$, this antenna is useful for integrating wireless sensor and communications devices onto and within systems.

\section{ACKNOWLEDGEMENT}

This work was supported by the NASA Integrated Vehicle Health Management (IVHM) program. The authors thank Nicholas C. Varaljay and Elizabeth A. McQuaid for fabricating the circuits.

\section{REFERENCES}

[1] D. Sievenpiper, R. F. J. Broas, and E. Yablonovitch, "Antennas on high-impedance ground planes," in IEEE Int. Micro. Symp. Dig., Vol. 3, June 13-19, 1999, Anaheim, CA, pp. 1245 - 1248.

[2] D. Sievenpiper, Lijun Zhang, R. F. J. Broas, N. G. Alexopolous, E. Yablonovitch, "High-impedance electromagnetic surfaces with a forbidden frequency band," IEEE Trans. Micro. Theory and Tech., Vol. 47, Iss. 11, pp. 2059 - 2074, Nov. 1999.

[3] D. Sievenpiper, "High-impedance electromagnetic surfaces," Ph.D. dissertation, Dept. Elect. Eng., Univ. California at Los Angeles, Los Angeles, CA, 1999.

[4] W. E. McKinzie III and R. R. Fahr, "A low profile polarization diversity antenna built on an artificial magnetic conductor," in IEEE Ant. and Prop. Society Int. Symp. Dig., 2002, pp. 762 - 765 vol.1.

[5] A. P. Feresidis, G. Goussetis, Shenhong Wang and J. C. Vardaxoglou, "Artificial magnetic conductor surfaces and their application to low-profile high-gain planar antennas," IEEE Trans. Ant. and Prop., Vol. 53, No. 1, pp. 209-215, Jan. 2005.

[6] A. Pirhadi, M. Hakkak, F. Keshmiri and R. K. Baee, "Design of compact dual band high directive electromagnetic bandgap (EBG) resonator antenna using artificial magnetic conductor," IEEE Trans. Ant. and Prop., Vol. 55, No. 6, pp. 1682-1690, June 2007.

[7] J. D. Shumpert, W. J. Chappell and L. P. B. Katehi, "Parallel-plate mode reduction in conductor-backed slots using electromagnetic bandgap substrates," IEEE Trans. Micro. Theory and Tech., Vol. 47, Iss. 11, pp. 2099-2104, Nov. 1999.

[8] H. Chu, Y. X. Guo, F. Lin and X. Q. Shi, "Wideband $60 \mathrm{GHz}$ onchip antenna with an artificial magnetic conductor," in IEEE Int. Symp. on Radio-Freq. Integration Tech. Dig., Dec. 2009, Singapore, pp. 307-310.

[9] G. Bianconi, S. Genovesi, A. Monorchio and G. Manara, "Investigation on the relation between dispersion diagram and the radiation pattern of low-profile antennas," in IEEE Ant. and Prop. Society Int. Symp. Dig., 2008.

[10] S. Nikolaou, D. E. Anagnostou, G. E. Ponchak, M. M. Tentzeris, and J. Papapolymerou, "Compact ultra wide-band (UWB) CPWfed elliptical monopole on liquid crystal polymer (LCP)," in IEEE AP-S Int. Symp. on Ant. and Prop.Dig., Albuquerque, NM, July 914, 2006, pp. 4657-4660.

[11] S. Nikolaou, N. D. Kingsley, G. E. Ponchak, J. Papapolymerou and M. M. Tentzeris, "UWB elliptical monopoles with a reconfigurable band notch using MEMS switches actuated without bias lines," IEEE Trans. on Ant. and Prop., Vol. 57, No. 8, Aug. 2009, pp. 2242-2251. 